\title{
MyoTrack: A 3D Deformation Field Method to Measure Cardiac Motion from Gated SPECT
}

\author{
Jean-Philippe Thirion ${ }^{1}$ and Serge Benayoun ${ }^{1}$ \\ ${ }^{1}$ HealthCenter Internet Services, 449 route des crêtes, \\ 06560 Sophia-Antipolis, France \\ jean-philippe.thirion@healthcenter.com
}

\begin{abstract}
We are presenting a new method for Gated SPECT analysis. We segment the myocardium walls in the first time frame (end diastole) and then compute volumetric motion fields between time frames for tracking. The application of dense motion field to segmented walls provides us with individual 3D trajectories for endo- and epicardium wall points. Besides traditional measurements like ejection fraction, radial motion and thickness variations, it allows us to derive original measurements such as focus of contraction, axial contraction, elevation and twist, and compute an average image where cardiac motion blur is suppressed. The method is packaged in a comprehensive research prototype, MyoTrackVB, which is currently evaluated by medical partners with more than 200 cases already processed.
\end{abstract}

\section{Introduction}

Many different imaging modalities have been used to study human heart motion: $\mathrm{Nu}-$ clear Medicine (first path, 2D and 3D blood pool, gated SPECT), MRI (Gated MRI, Tagged MRI, Phase contrast MRI), Gated CT, Ultrasound, angiography.... SPECT imagery suffers from low resolution and high level of noise in the images and medical researchers interested in heart motion analysis generally disregard Gated SPECT to the benefit of other more contrasted but most costly or invasive modalities. However, from a clinical standpoint, Gated SPECT is one of the most widespread exam for wall motion analysis. It can be performed simultaneously to a Myocardial Perfusion Scintigraphy (MPS) study without much additional cost. One advantage is that wall motion information is obtained in the same reference frame than perfusion. Our method, MyoTrack, is the adaptation of a generic volumetric motion field technique (see [16]) to the case of Gated SPECT images and is based on tracking. In contrast, the majority of existing Gated SPECT techniques are based on independent segmentations of time frames or measurements dedicated to very peculiar indices such as the "ejection fraction" (EF). In that sense, we are much closer to methods developed for MRI or CT. We describe first the physical particularities of Gated SPECT as well as the clinically relevant measurements used to analyze Cardiac Motion. We summarize the principles of existing analysis methods. We then describe MyoTrack and explain how physical constraints of Gated SPECT are taken into account. Finally, we present quantitative results obtained from diseased and normal patients.

S.L. Delp, A.M. DiGioia, and B. Jaramaz (Eds.): MICCAI 2000, LNCS 1935, pp. 697-706, 2000.

(C) Springer-Verlag Berlin Heidelberg 2000 


\section{Specificity of Gated SPECT Image Sequences}

Gated SPECT is a 3D Computed Tomography technique where acquisition is gated with the ECG. As images are obtained by averaging a lot of cardiac cycles, the image sequence is cyclic, first frame being the successor of the last one. It is measuring photon emitted by the destruction of radionuclides whose concentration can be considered as constant during the acquisition and proportional to the perfusion of the heart muscle (mainly the myocardium of the left ventricle). The heart being a muscle, it is incompressible which means that the divergence of the deformation field is null within the myocardium. As intensity in the images is proportional to perfusion, it means that we should measure constant intensity within the myocardium.

One major drawback of Gated SPECT is that the acquisition device has a large point spread function, which means limited resolution but also changes in image maxima when the myocardium is contracting: a larger wall appears brighter in the image. A normally perfused myocardium has a uniform perfusion distribution, but unfortunately diseased patients are generally presenting large perfusion defects where very little information is available. As noise level is high and as extra activities in bowels or liver can blend with the myocardium, automatic segmentation is really challenging (Figure 1).
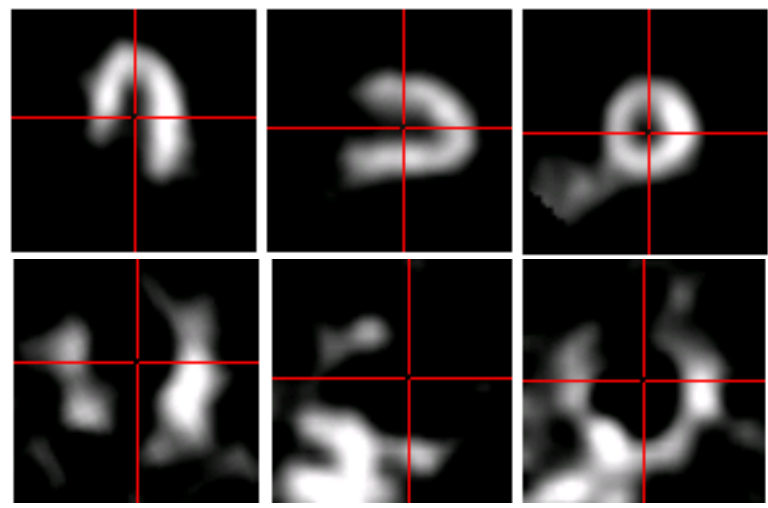

Figure 1: Horizontal long, vertical long and short axes of a normal subject (top) and a diseased subject (bottom)

\section{Existing Methods for Cardiac Dynamic Analysis}

One useful clinical index is the Ejection Fraction or EF, which is the ratio of the difference between End Diastolic Volume (EDV) and End Systolic Volume (ESV), divided by the EDV. The Stroke Volume (SV) is EDV-ESV. A lot of clinical papers are dealing with semi-automatic or automatic determination of EF (see for example 
[1],[2],[3]). Other useful clinical measures are radial motion and wall thickening (see [4],[5],[6]). Existing Gated SPECT analysis techniques can be classified into physical measurement of wall boundary positions, and into evaluation of mid-wall and wall thickness. Most methods rely on a frame by frame analysis based on independent segmentation of the myocardium. In [5] temporal analysis of intensity variations is related to width variations by empirical studies on point spread function effects. MyoTrack is based on tracking and is using comprehensively the whole sequence. It is much closer to methods developed for other modalities such as Gated MR ([7]), Tagged MR ([8], [9],[10]), Phase Contrast MR ([11]), Gated CT ([12],[13]), echocardiography ([14]) or PET ([15]). Tracking methods can be classified into 3D surfacebased tracking and 3D dense motion field computation. MyoTrack is closer to motion field methods that we are now detailing.

The work of Song and Leahy [12] is the application of 2D+T optical flow techniques to $3 \mathrm{D}+\mathrm{T}$ and is heavily relying on fluid mechanics. Two constraints are of particular interest to our problem, which are the conservation equation and the incompressibility constraint. The method is iterative (conjugate gradient descent) and solve for the two constraints (conservation and incompressibility) as well as a smoothness constraint. It is applied to Gated CT images. In the same spirit, Benayoun and Ayache [13] are measuring $3 \mathrm{D}$ dense deformation field. Aside from smoothness, the other constraint is the tracking of differential singularities. Although it is less physical than density conservation, it allows providing a better matching with respect to motion in the direction tangent to object boundaries. The cardiac application is also 3D Gated CT (dog heart). In the work of Klein [15], conservation and smoothness are solved for and the method is applied to 3D Gated PET images.

\section{MyoTrack, a New Method for Gated SPECT Analysis}

We have designed an iterative and multi-resolution generic method of motion field computation described in [16]. We have shown that 3D deformation computation can be also viewed as the "diffusion" of a deformable model through the contours of a reference object and that image driven constraints as simple as inward/outward unitary displacements along normals to contours, determined from tissue characterization, is sufficient to perform matching. We have also shown that image smoothing applied to the $3 \mathrm{D}$ deformation fields is an effective way to perform regularization. In [16], we have briefly described brain matching and gated SPECT analysis as potential applications, with some feasibility results. Since then, we have refined our technique in order to achieve a real clinical application for gated SPECT analysis that we call MyoTrack and which is presented here.

\subsection{General Principle}

The general principle of MyoTrack is to separate 3D motion computation from myocardium segmentation (Figure 2). The myocardium is segmented in the first image of 
the Gated SPECT sequence (generally the end diastole time frame) and then tracked throughout the whole sequence. The segmentation is based on template matching. The interest is twofold: first, it allows performing inter-patients comparisons as well as to design reference population models. It also helps providing a-priori knowledge for specific case interpretation as any pre-computation performed in the template's shape can be transferred to the specific case.

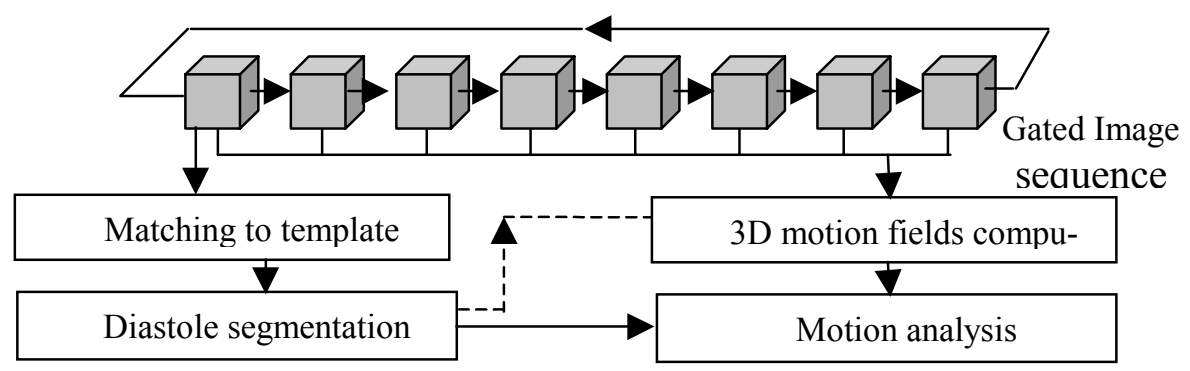

Figure 2: General principle. Segmentation and 3D image tracking are separated

\subsection{Myocardium Segmentation for End Diastole Time Frame}

Segmentation of the myocardium is a 4-steps method:

1. During the initialization phase an affine transformation is found between the end diastole image and a reference template shape. We are using an Iterative Closest Point method (ICP, see [17]) and are searching for an affine transformation. Two 3D deformable parameterized ellipsoids are defined, once for all in the template's reference frame.

2. The coupled ellipsoids are iteratively deformed to match to the edge points as well as to ensure smoothness constrains and anatomical a-priori constraints (for example, myocardium approximate width conservation). Sample points for regular angular subdivision in polar coordinates are used to represent deformed ellipsoids.

3. The base hole is segmented using a deformable 2D contour in polar representation.

4. A $3 \mathrm{D}$ closed surface representation in Cartesian coordinates is computed from the radius maps of endo- and epicardium positions and base definition.

\subsection{Dense Motion Field Computation}

We only describe here the adaptation necessary from [16], which are intensity conservation, cyclicity, incompressibility and adaptive rigidity constraints. The output is, for each time frame, the node positions of a deformed 3D grid. The reference is a regular 3D grid defined in end diastole time frame. The method is iterative and multiresolution, ending with a resolution as thin as voxel sizes. 
1. Intensity conservation is ensured by a formula derived from optical flow and used for motion computation. $\mathrm{s}$ and $\mathrm{m}$ are the respective intensities in the reference and model images and $(m-s)^{2}$ is used to avoid singularities when $\vec{\nabla} s$ is small: $\vec{v}=\frac{(m-s) \vec{\nabla} s}{(\vec{\nabla} s)^{2}+(m-s)^{2}}$

2. To ensure cyclicity as well as regularity through time, we are restricting at each iteration the $x(t), y(t)$, and $z(t)$ displacements of each node of the deformable grid to first harmonics.

3. Incompressibility corresponds to null divergence of the deformation field. We are measuring, at each iteration, the volume element of each cell of the grid (within the Myocardium). As the grid in the end diastole image is regular, this volume should remain one voxel cube. Homothetic variations are applied as additional constraints to make cell volumes tend toward unit values.

4. Our rigidity constraint is adaptive. The endocardium cavity is much more deformable than epicardium boundary thanks to the use of adaptive filtering to regularize deformation fields.

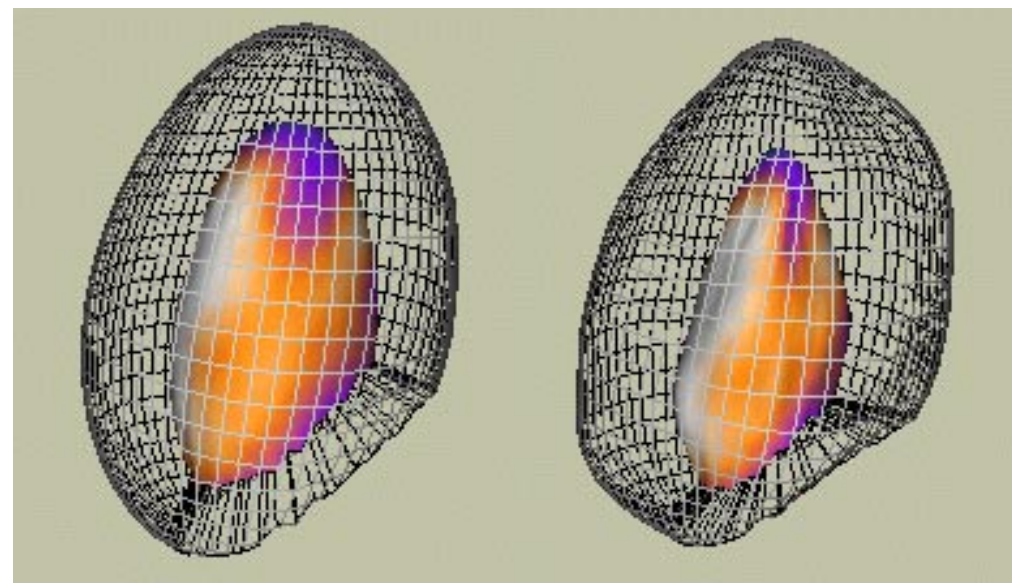

Figure 3: End diastole and end-systole surface representation (endo- and epicardium), with perfusion information.

\subsection{Dynamic 3D Surface Representations}

As they are defined from a regular grid in the end diastole frame, deformation fields can be applied to 3D surface representation of the myocardium walls. Each vertex of the surface representation follows a 3D closed trajectory deduced from the 3D deformed grids via tri-linear interpolation within the grid cells (see Figure 3). On the contrary to all traditional methods of gated SPECT analysis, and much closer to Gated MR or CT techniques, we are able to analyze other aspects than simply surface radial motion. 


\section{Motion Analysis Methods}

MyoTrackVB has various outputs which are: dynamic 3D surface representation of the myocardium walls in Cartesian coordinates, dynamic Bull's eye representations (or polar maps) of wall positions and perfusion, measures obtained from dense 3D deformation field for all time frames....

\subsection{Dynamic 3D Surfaces, EF,...}

Dynamic 3D surface representations can be manipulated and visualized using MyoTrackVB (see Figure 3) and convey important clinical information about myocardium function. To get more tractable information, we can also compute the endocardium volume for each time frame, which is giving EDV, ESV, SV and EF values. The "Focus of Contraction" (FOC) is intermediate between full dynamic surface data and EF value. We define the FOC as the locus which minimizes, in a least square sense, the distance to all lines supporting instantaneous velocity vectors. We found that the focus of contraction trajectory is very compact and situated near the center of the myocardium "equator" (see results section).

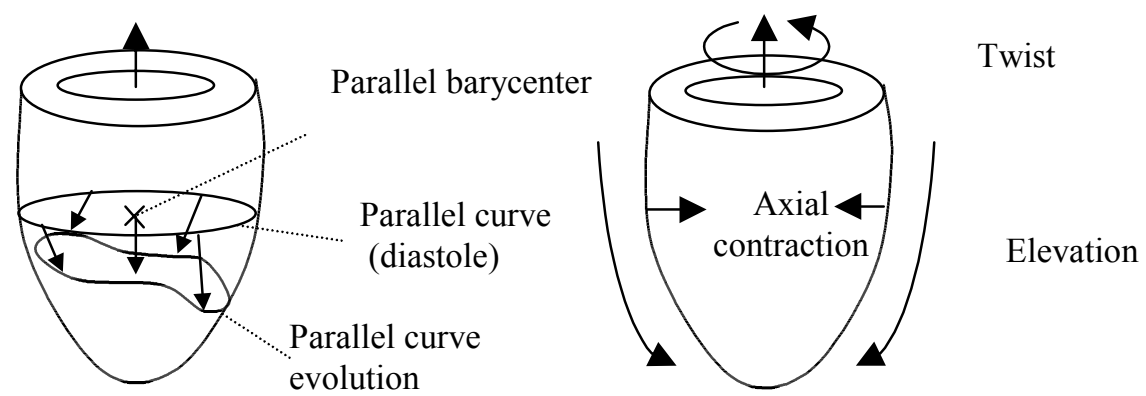

Figure 4: evolution of parallel lines to measure axial contraction, elevation and twist

MyoTrackVB can present also the amplitude and phase of radial motion and thickness variations in Bull's eye representation (Figure 5). Contrary to other Gated SPECT methods, each vertex of the surface representation is free to move in $3 \mathrm{D}$, except for regularization constraints, which is changing definitions such as width variation which becomes the evolution of the distance between identified pairs of points instead of surface motion. 3D trajectories allow us to study other motion, such as axial contraction, elevation and twist. To define more precisely such quantities (see Figure 4), we are considering the evolution of "parallels", that is, sets of points having the same angle $\theta$ in polar coordinates in end diastole image and forming a closed curve. Parallel curve barycenter motion along the axis of the myocardium is the elevation, the square root of the surface variation of the minimal surface defined by the curve gives the axial contraction ratio and the "rotation" of the curve around the myocardium axis 
gives the twist component. These values are defined for all "latitudes" $\theta$ ranging from $-\pi / 2$ to $+\pi / 2$.

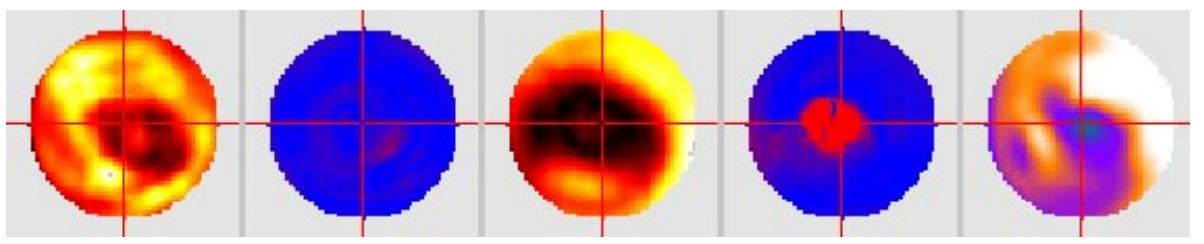

Figure 5: Left to right, thickness variation amplitude and phase, radial motion amplitude and phase, and perfusion.

\subsection{Using the Dense Motion Field Computation}

To reduce noise level, all time frames are summed to give a single static representation for MPS studies. However, cardiac motion blurs the image, which reduces the signal. As suggested in [16] or [15], we are using the computed motion field to compensate for cardiac motion to get a static image of the heart with a much higher contrast to noise ratio (see Figure 6). The deformation fields are applied to all time frame images to make them superimposable to the end diastole image, and all deformed images are then summed. This operation is especially useful in the case of low count data.
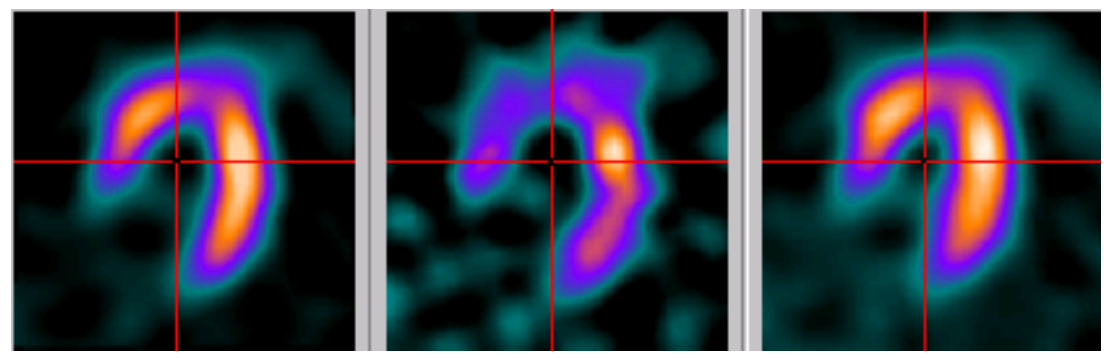

Figure 6: Diastole frame (middle), direct average of 8 time frames (right), average with motion compensation (left).

\section{Results}

We have performed several validation experiments of the Ejection Fraction computation with different medical partners. The Hôpital Henri Mondor has performed a blind validation on 41 severely diseaded patients, imaged two times with Thallium, at 20 minutes and at 4 hours (see [18]). Comparison is performed with ERNA (2D blood pool) and QGS ([2]). St Luke's Hospital, New York, as performed a blind validation with 57 single head MIBI data, with comparison with ERNA and with SPECT EF. We have also performed a validation (non-blind) with Emory University Hospital on 22 cases with comparison with gated MRI and with QGS. EF correlation between 
MyoTrack and other methods are ranging between $80 \%$ and 95\%. In total, MyoTrack has been run fully automatically on more than 200 cases without major failure of segmentation or tracking (visual assessment with contours overlay). We are presenting results for 5 normal cases with a normal angiogram and good perfusion and 5 diseased cases with a severe infarct and visible perfusion deficits. The aim is to compare different outputs of MyoTrack in various conditions in order to evaluate, beside EF computations, which measurements has to be investigated with respect to further clinical validation.

With respect to Focus of contraction, a first interesting observation is that, for all cases (normal as well as abnormal) the trajectory of the FOC is a double loop, the larger one being covered during diastole and the smaller one during systole. We have computed the barycenter of each FOC trajectory as well as the average distance to the barycenter (compactness) and the Residual Mean Square (RMS) distance between the instantaneous velocity direction lines and the FOC, normalized using EDVs (focalization).

For Axial contraction, elevation and twist 1D profiles, we are presenting these profiles in Figure 7. The pattern is more coherent for normal than for diseased subjects. The clinical usefulness of these indices has still to be evaluated. It might be that, thanks to perfusion defects, the twist motion could be retrieved with more accuracy for diseased than for normal cases, which would be troublesome for clinical application, but the two other indices are very promising.

\section{Conclusion}

We have developed a new technique for the interpretation of Gated SPECT image sequences based on flow field techniques and inspired from methods developed for other kind of modalities such as Gated MRI or Gated CT. We have packaged the whole method into a fully automated research prototype called MyoTrackVB. MyoTrackVB is taking as input raw tomograms and has a large variety of possible outputs: average image with Cardiac motion blur compensation, 3D dynamic surfaces interactive display, amplitude and phase Bull's eyes for radial motion and thickness, axial contraction, elevation and twist 1D profiles, focus of contraction and Ejection Fraction and endocardium volumes (EDV, ESV) computations. These measures are now studied in collaboration with medical partners to determine their clinical relevance. The automation of the processing has already permitted to process more than 200 cases. 

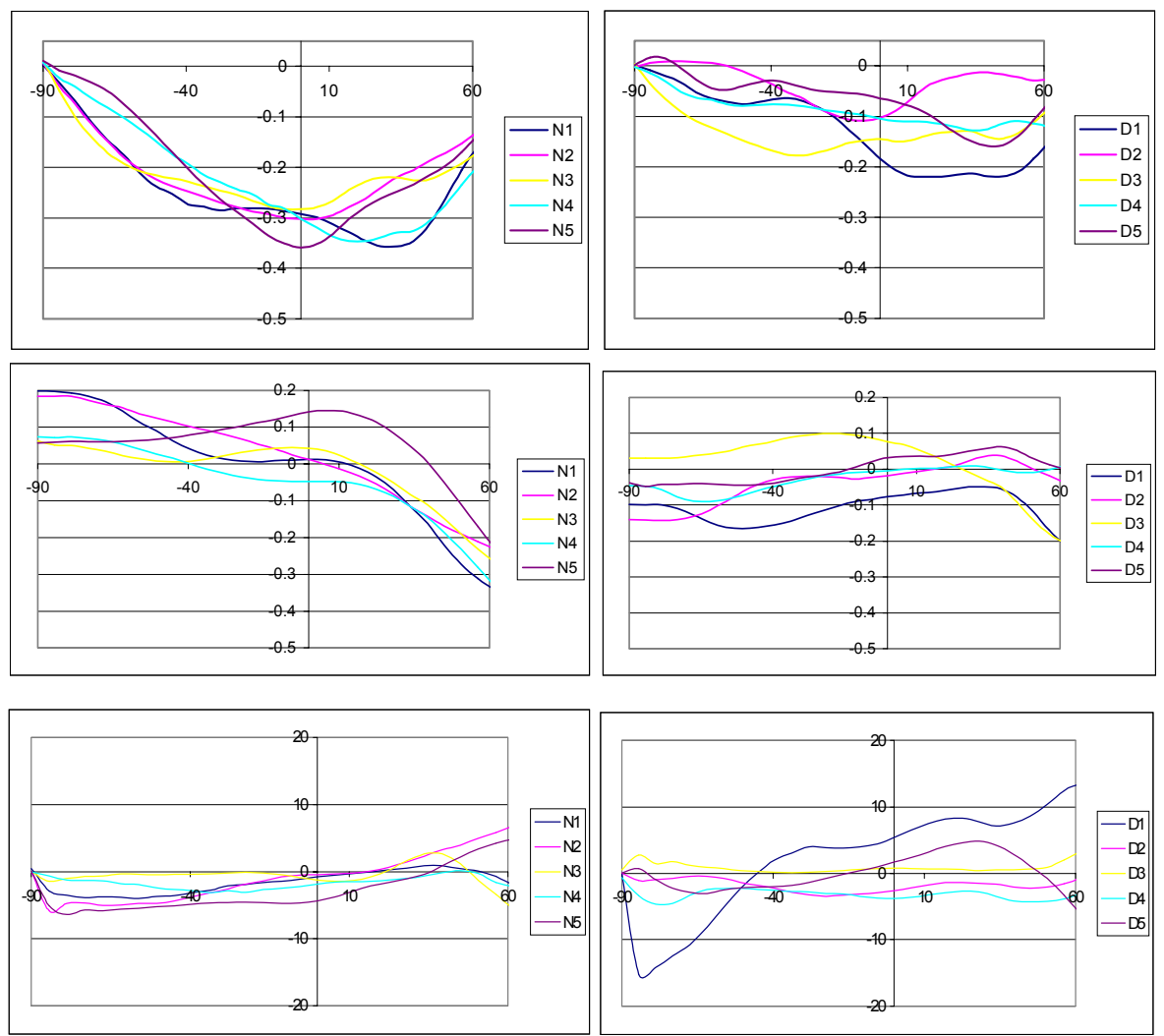

Figure 7: Top to bottom: axial contraction, elevation and twist for (left) normal subjects and (right) disease subjects.

\section{Acknowledgments}

Thanks to Drs. M. Meignan, J. Rosso and E. Itti from Hôpital Henri Mondor, Creteil, France, Drs. G. Depuey and M. Kamran from St'Lukes Hospital, New York, USA and Dr. J. Vansant and Mr. M. Blais from Emory University Hospital, Atlanta, USA, for collaborating with us on EF validation, and to Dr. Martin Kernberg, from the Department of Medicine, UCSF, California, and Dr. Michael Goris, Stanford University, for stimulating discussions about cardiac anatomy and SPECT.

\section{References}

1. E.G. Depuey, K. Nichols, and C. Dobrinsky, "Left ventricular ejection fraction assessed from gated technetium-99m-sestamibi SPECT", J. of Nuclear Medicine, vol. 34, no. 11, pp 1871--1876, November 1993.

2. Germano, H. Kiat, P.B. Kavanaugh, M. Moriel, M. Mazzanti, and H.T. et al. Su, “Automatic quantification of ekection fraction from gated myocardial perfusion spect" The Journal of Nuclear Medicine, vol. 36, pp. 2138--2147, 1995. 
3. H. Everaert, P.R. Franken, M. Goris, A. Momen, and A. Bossuyt, "Left ventricular ejection fraction from gated SPECT myocardial perfusion studies: a method based on radial distribution of count rate density across the myocardial wall" European Journal of Nuclear Medicine, vol. 23, no. 12, pp. 1628--1633, December 1996.

4. M.L. Goris, C. Thompson, L.J. Malone, and P.R. Franken, "Modelling the integration of myocardial regional perfusion and function" Nuclear Medicine Communication, vol. 15, pp. 9--20, 1994.

5. C.D. Cooke, E.V. Garcia, S.J. Cullom, T.L. Faber, and I.P. Roderic, 'Determining the accuracy of calculating systolic wall thickening using a fast Fourier transform approximation: A simulation study based on canine and patient data", The Journal of Nuclear Medicine, vol. 35, no. 7, pp. 1185--1192, July 1994.

6. I. Buvat, M.L. Bartlett, A.N. Kitsiou, V. Dilsizian, and S.L. Bacharach, "A "hybrid" method for measuring myocardial wall thickening from gated pet/spect images," The Journal of Nuclear Medicine, vol. 38, no. 2, pp. 324--329, February 1997.

7. L.H. Staib and J.S. Duncan, "Model-based deformable surface finding for medical images," IEEE Trans. on Medical Imaging, vol. 15, no. 5, pp. 720--731, October 1996.

8. T.S. Denney and J.L. Prince, "Reconstruction of 3-d left ventricular motion from planar tagged cardiac MR images: An estimation theoretic approach," IEEE Trans. on Medical Imaging, vol. 14, no. 4, pp. 625--635, December 1995.

9. J. Park, D. Metaxas, A.A. Young, and L. Axel, "Deformable models with parameter functions for cardiac motion analysis from tagged MRI data," IEEE Trans. on Medical Imaging, vol. 15, no. 3, pp. 278--289, June 1996.

10. A.A. Amini, Y. Chen, R.W. Curwen, V. Mani, and J. Sun, "Coupled b-snake grids and constrained thin-plate splines for analysis of 2-d tissue deformation from tagged mri," IEEE Trans. on Medical Imaging, vol. 17, no. 3, pp. 344--355, June 1998.

11. F.G. Meyer, R.T. Constable, A.J. Sinusas, and J.S. Duncan, "Tracking myocardial deformation using phase contrast MR velocity fields: A stochastic approach," IEEE Trans. on Medical Imaging, vol. 15, no. 4, pp. 453--465, August 1996.

12. S.M. Song and R.M. Leahy, "Computation of 3-d velocity fields from 3-d cine CT images of a human heart," IEEE Trans. on Medical Imaging, vol. 10, no. 3, pp. 295--306, September 1991.

13. S. Benayoun and N. Ayache, "Dense non-rigid motion estimation in sequences of medical images using differential constraints," International Journal of Computer Vision, vol. 26, no. 1, pp. 25--40, January 1998.

14. F.H. Sheehan, M.S. Bolson, R.W. Martin, G. Basheim, and J. McDonald, "Quantitative three-dimensional echocardiography: Methodology, validation, and clinical application," in MICCAI'98, October 1998, vol. 1496 of Lecture Notes in Computer Science, pp. 102--109, Springer.

15. Gregory J. Klein, "Forward deformation of pet volumes using material constraints," in IEEE Workshop on Biomedical Image Analysis (WBIA'98), B. Vemuri, Ed., Santa Barbara, California, June 1998, pp. 64--71, IEEE.

16. Jean-Philippe Thirion, "Image matching as a diffusion process: an analogy with Maxwell's demons," the journal of Medical Image Analysis (MEDIA), vol. 2, no. 3, pp. 243--260, 1998.

17. J. Declerck, J. Feldmar, M.L. Goris, and F. B etting, “Automatic Registration and Alignment on a Template of Cardiac Stress $\backslash \&$ Rest SPECT Images," in Mathematical Methods in Biomedical Image Analysis, June 1996.

18. E. Itti, J. Rosso, M. Meignan, S. Benayoun and J-P. Thirion, "MYOTRACK: a new method to measure ejection fraction from Gated SPECT: preliminary evaluation of precision and accuracy with 201Tl”, American Society of Nuclear Medicine SNM'2000, saint Louis, USA. 